\title{
UTILITY OF COMPARATIVE ANCHOR-TAGGED SEQUENCES AS PHYSICAL ANCHORS FOR COMPARATIVE GENOME ANALYSIS AMONG THE CULICIDAE
}

\author{
ERIC W. CHAMBERS, DIANE D. LOVIN, AND DAVID W. SEVERSON \\ Center for Tropical Disease Research and Training, Department of Biological Sciences, University of Notre Dame, \\ Notre Dame, Indiana
}

\begin{abstract}
The development of comparative genetic maps in multiple species of mosquitoes could prove extremely useful in the search for those genes that contribute to mosquito vector competence or genes associated with other phenotypes of interest. To effectively compare these gene maps, markers must be developed that are based on chromosomal regions conserved throughout the Culicidae. We designed 35 polymerase chain reaction (PCR) primer pairs based upon orthlogous exons in Aedes aegypti and Drosophila melanogaster or Anopheles gambiae. Twenty-three of the primers yielded a single PCR product in at least one dipteran, in addition to Ae. aegypti, when screened with genomic DNA from seven dipterans, including five mosquito species. Eight of the primers amplified a single PCR product in only Ae. aegypti, while four primer pairs gave no PCR product in any species. The 23 successful comparative anchor-tagged sequence primer pairs give broad genome coverage in Ae. aegypti, and more importantly demonstrate an efficient strategy for developing comparative anchor marker loci for any species of Culicidae.
\end{abstract}

\section{INTRODUCTION}

The family Culicidae contains approximately 3,500 species of mosquitoes, ${ }^{1}$ and historically most genetic research efforts have been directed towards the two principal disease vector organisms, Aedes aegypti and Anopheles gambiae. The first detailed genetic map for Ae. aegypti consisted of 77 morphologic, isozyme, and insecticide resistance markers. ${ }^{2}$ Early efforts to produce genetic maps of the Anopheles spp. were less successful due to a dearth of morphologic and isozyme markers, along with the difficulty of rearing these mosquitoes in the laboratory. In addition, within both the Culicinae and Anophelinae, these markers were not applicable for detailed genome coverage in a single mapping population and were often non-neutral in nature. Despite the difficulty of working with such markers, genetic linkage maps were constructed for Ae. togoi, Ochlerotatus triseriatus, Ae. aegypti, An. albimanus, An. quadrimaculatus, and An. gambiae. ${ }^{3,4}$ Furthermore, a limited comparison of isozyme loci suggested a high degree of synteny. ${ }^{5}$ Synteny refers to loci that remain physically linked in different species.

The advent of DNA-based genetic markers including restriction fragment length polymorphisms (RFLPs), random amplified polymorphic DNA (RAPD), single-strand conformation polymorphisms (SSCPs), and microsatellites has allowed the construction of detailed linkage maps for a small number of mosquito species. The first such map, in Ae. aegypti, was generated using RFLP markers ${ }^{6}$ and consisted of 50 DNA markers based mostly on random cDNAs that identified 53 loci across the three linkage groups. The most recent map for this species consists of 146 markers across $205 \mathrm{cM}^{7}$ Two RAPD-SSCP linkage maps for Ae. aegypti were also constructed, the first consisted of 96 loci that covered 168 $\mathrm{cM}^{8}$, while the second consisted of 94 SSCP markers based upon single nucleotide polymorphisms (SNPs) that covered $134 \mathrm{cM}^{9}$ Within the anophelines, a DNA-marker-based genetic map of An. gambiae was constructed using 148 microsatellite markers covering $215 \mathrm{cM}$. $^{10}$ These genetic maps have proven useful within Ae. aegypti and An. gambiae in efforts to identify genomic regions or genes associated with mosquito competence to transmit a variety of pathogens. ${ }^{11-16}$

Of interest is the construction of detailed genetic linkage maps in a greater number of mosquito species. Comparative
RFLP-based linkage maps for the mosquitoes Culex. pipiens, ${ }^{17}$ Ae. albopictus, ${ }^{18}$ Oc. triseriatus (formerly Aedes),${ }^{19} \mathrm{Cx}$. tritaeniorhynchus, ${ }^{20}$ and Armigeres subalbatus ${ }^{21}$ were previously constructed using Ae. aegypti cDNA clones as probes to Southern blots. This strategy was successful because many $A e$. aegypti cDNA sequences are highly conserved across mosquito species. $^{22}$ These maps allowed for the direct comparison of these species because the markers are based upon expressed genes (Type I markers) as opposed to microsatellites (Type II markers); that is, although microsatellites have proven useful in developing gene maps in many organisms, they are less useful when making comparisons between organisms because they are usually not conserved between species. $^{23}$

Despite the success in constructing comparative gene maps for several mosquito species using RFLP markers, there has been no attempt to develop polymerase chain reaction (PCR)-based DNA markers that can be used across species within the Culicidae. The utility of orthologous gene comparisons for development as tools for PCR-based identification of conserved syntenies has been demonstrated for both birds and mammals. ${ }^{23-25}$ Markers developed in this manner have been referred to as comparative anchor-tagged sequences (CATS) and have been used to generate comparative genome maps in the human, dog, and horse..$^{26-28}$ The identification of such a set of markers within mosquitoes could prove to be extremely useful among those species that although not genetically well characterized, nonetheless are of great importance medically.

In this study, we investigated the utility of the CATS marker strategy for developing comparative genome maps among mosquito species. We have evaluated 35 putative CATS primer sets across six mosquito species and two more distant species within the Diptera. Twenty-three of these CATS amplified a single PCR product in at least one dipteran species in addition to Ae. aegypti. This simple strategy in which orthologous exons of two or more dipterans are identified, sequence alignments are made, and PCR primers are designed for highly conserved regions should facilitate the rapid identification of PCR-based genetic markers that provide physical anchor loci with broad application to genetically uncharacterized mosquito species and perhaps more distant dipteran species as well. 


\section{MATERIALS AND METHODS}

Candidate selection for CATS. Identity searches were conducted on 71 previously mapped, partial cDNA clones or known genes from adult $A e$. aegypti Liverpool females ${ }^{7}$ by comparing nucleotide sequences against both the nonredundant as well as the non-human, non-mouse expressed sequence tag (EST) databases using the BLASTN computer program. ${ }^{29}$ Thirty-five of the Ae. aegypti sequences showed significant nucleotide identities, based upon an expected value (E) of less than $\mathrm{e}^{-15}$ as our critical value for defining sequences as orthologs with sequences from Drosophila melanogaster and/or An. gambiae. Nucleotide sequences from the putative orthologs were aligned using the Multiple Alignment Program (MAP) on the Baylor College of Medicine (BCM) Search Launcher web site. ${ }^{30}$

Primer design. Primers were designed using the PRIMER program (Whitehead Institute, Massachusetts Institute of Technology, Cambridge, MA). We first attempted to design primers within regions of the sequences that exhibited complete nucleotide sequence identity between Ae. aegypti, D. melanogaster, and An. gambiae. In most instances, however, we designed primers to contain no more than 2-3 mismatches with complete sequence identity for the final 5-6 nucleotides at the $3^{\prime}$ end. When mismatches were unavoidable, we biased nucleotide selection to the Ae. aegypti sequence. We designed primers to be 18-22 nucleotides in length with a $\mathrm{G}+\mathrm{C}$ content of approximately $50 \%$. When possible, we placed a GC clamp at the $3^{\prime}$ end. The expected PCR product size was between 100 and 400 bases. Whenever possible, we avoided selecting primers with the $3^{\prime}$ nucleotide representing the third base position in a codon.

Isolation of DNA. Isolation of DNA from individuals representing Ae. aegypti, Oc. triseriatus, Ae. togoi, An. gambiae, $A r$. subalbatus, and D. melanogaster were performed as previously described. ${ }^{31}$ Genomic DNA representing individuals from $C x$. pipiens was kindly provided by Dr. Akio Mori and that from Rhagoletis pomonella was kindly provided by Dr. Jeff Feder (both at the University of Notre Dame, Notre Dame, IN).

Primer optimization. The PCRs were conducted in 96-well plates using a Hybaid (Ashford, Middlesex, United Kingdom) Omnigene thermal cycler. Each $25-\mu \mathrm{L}$ PCR mixture contained $1 \times$ Taq buffer $(10 \mathrm{mM} \mathrm{KCl}, 2 \mathrm{mM}$ Tris, $\mathrm{pH} 9.0,0.02 \%$ Triton X) 1.5 or $3.0 \mathrm{mM} \mathrm{MgCl}_{2}, 0.4 \mathrm{mM}$ each of dATP, dCTP, dGTP, and dTTP, 5-10 pmoles of each primer, 1 unit of Taq DNA polymerase, and $5 \mathrm{ng}$ of genomic DNA. Six primer sets were screened with eight dipteran species per plate. We screened each primer set at three anneal temperatures, $50^{\circ} \mathrm{C}$, $54^{\circ} \mathrm{C}$, and $58^{\circ} \mathrm{C}$. The standard PCR conditions were $94^{\circ} \mathrm{C}$ for five minutes, followed by 30 cycles at $94^{\circ} \mathrm{C}$ for one minute, an annealing step at one of the three previously mentioned temperatures for one minute, $72^{\circ} \mathrm{C}$ for two minutes, and a final extension at $72^{\circ} \mathrm{C}$ for 10 minutes. Primer sets with an expected PCR product size less than 200 basepairs were subjected to an alternate set of PCR conditions: $94^{\circ} \mathrm{C}$ for 10 minutes, followed by 40 cycles at $94^{\circ} \mathrm{C}$ for 20 seconds, annealing at one of the three temperatures for 20 seconds, $72^{\circ} \mathrm{C}$ for 30 seconds, and a final extension at $72^{\circ} \mathrm{C}$ for 10 minutes. The PCR products were size fractionated by electrophoresis in $3 \%$ agarose gels, stained with ethidium bromide, and visualized under UV light.
Sequencing. Amplified PCR products were spin-column purified using the QIAquick PCR purification kit (Qiagen, Valencia, CA). The purified PCR products were then quantified visually after electrophoresis on $3.0 \%$ agarose gels. The PCR product templates were subjected to cycle sequencing using the ABI Prism Big Dye Terminator kit according to the suppliers recommendations (Applied Biosystems, Inc., Foster City, CA) and an ABI Prism 310 Genetic Analyzer. Singlepass sequence information was obtained from each strand of each PCR product. Sequence data were then subjected to BLASTN and BLASTX searches of the GenBank nonredundant and EST databases using default settings, ${ }^{29}$ as well as pairwise sequence alignment to the original Ae. aegypti clone sequence using the Blast2 program. ${ }^{32}$

Linkage map. The linkage map was drawn using the DrawMap computer program. ${ }^{33}$ Map distances are reported in Kosambi centiMorgans.

\section{RESULTS}

From an initial screening of 71 Ae aegypti cDNAs, we designed PCR primers for 35 putative CATS. Of the 36 cDNAs for which no primers were designed, 15 showed insufficient nucleotide identity with D. melanogaster or An. gambiae, based on our primer design criteria, to allow amplification of a minimum fragment size of at least 100 basepairs, while the remaining 21 Ae. aegypti cDNAs showed no nucleotide identity with either D. melonogaster or An. gambiae when screened against either the non-redundant or nonhuman non-mouse EST databases using the BLASTN program.

The 35 CATS primer sets were used to screen genomic DNA from six mosquito species including Ae. aegypti, Oc. triseriatus, Ae. togoi, An. gambiae, C. pipiens, and Ar. subalbatus, as well as two more distant species within the dipterans, $D$. melanogaster and $R$. pomonella. Twenty-three primer pairs (Table 1) yielded a single PCR product with at least one species in addition to Ae. aegypti, while 8 primer pairs produced a single PCR product only in Ae. aegypti. Four of the primer sets failed to produce a PCR product in all of the eight dipterans screened, including Ae. aegypti. Two typical CATS primer screening results are seen in Figure 1. The genetic locations in $A e$. aegypti ${ }^{7}$ of all CATS for which we successfully obtained a PCR product in at least one non-Ae. aegypti species are shown in Figure 2.

We observed varying rates of success for amplification of individual CATS within each of the eight species tested (Table 2). Amplification of a single PCR product varied among species relative to Ae. aegypti with a low of $13 \%$ seen in $R$. pomonella and a high of $60 \%$ seen in Oc. triseriatus. Thirteen of the twenty-three CATS primer pairs (56\%) amplified with four or more of the species, while the remaining 10 CATS primer pairs (44\%) amplified between two to three different species.

We selected three CATS markers, LF178, LF158, and LF103, for sequence analysis and identity verification. They were selected as random representatives of each of the three Ae. aegypti linkage groups. Also, these CATS amplified a single PCR product in at least five of the eight species screened. We sequenced the PCR products from these CATS following amplification of genomic DNA from Ae. aegypti, $A$. subalbatus, and/or D. melanogaster. 
TABLE 1

Comparative anchor-tagged sequence (CATS) primers and PCR conditions*

\begin{tabular}{|c|c|c|c|c|}
\hline CATS no. & Accession no. & $\begin{array}{c}\text { PCR conditions } \\
\text { Annealing temperature, } \\
{ }^{\circ} \mathrm{C} / \mathrm{mM} \mathrm{Mg}+\end{array}$ & $\begin{array}{l}\text { Predicted product } \\
\text { size (bp) }\end{array}$ & Primer sequences 5 '-forward/reverse- $3^{\prime}$ \\
\hline LF90 & T58320 & $50 / 1.5$ & 110 & GAAGAGGTGCAGGTCTCGCT/ACAGATCCGTGACATGGACG \\
\hline LF92 & BM005493 & $55 / 1.5$ & 277 & CCCAGATCAGCAGCGTTTG/TTACTTCAGCTTCTTCTTCGGG \\
\hline LF96 & BM005491 & $50 / 1.5$ & 164 & ATCAAGCTCGCCAAGGTC/GGGTTGTAGACGGCGTTG \\
\hline LF99 & BM005477 & $54 / 1.5$ & 356 & AAGCGCAAGCACAAGAAG/TTGATCGACTTGTTCAGCT \\
\hline LF103 & BM005488 & $54 / 1.5$ & 181 & ATGCGCGATCTTCGGATC/AGTGGACGGCAATCTTTTCG \\
\hline LF106 & BM005490 & $54 / 1.5$ & 175 & CAAAGCAGCCGCAAAAGA/GGGTGATCAGATTGTAGG \\
\hline LF108 & T58322 & $50 / 1.5$ & 265 & AAGTCCGGCAAGCTGAAGG/GCTTGATCTGTTCCAGGGC \\
\hline LF124 & BM005518 & $54 / 1.5$ & 103 & GAGGAGAAGCGCAAGCAC/TTGGGCATGGCTGAACAC \\
\hline LF129 & BM005504 & $54 / 3.0$ & 300 & GCACCAGGAAAGTCGGAATC/AGTACACCCAGGCACCGC \\
\hline LF138 & T58332 & $54 / 1.5$ & 204 & CGTGTTCAACTACGACAT/TCCTTCAATGTACGATGAG \\
\hline LF158 & BM005485 & $50 / 3.0$ & 239 & GGAAGGGTCTGAAGATCACC/AGCACTTCCTTGCAGGTACC \\
\hline LF168 & R47184 & $54 / 1.5$ & 113 & GGAGGACGCTGCAAGCAC/ACGATGTTCCGGATCACG \\
\hline LF178 & T58309 & $50 / 1.5$ & 207 & GCCGTGGTGTCCCAGATC/GCAGACGCATACGGATGTG \\
\hline LF179 & BM005479 & $50 / 3.0$ & 234 & AACAAAGTGACCCAACTGACTG/ACACCTTGACATTGGCCTTG \\
\hline LF188 & BM005472 & $54 / 1.5$ & 208 & CTTTGACGACTTCAAGCGC/ACGGAAGCGAAGGCC \\
\hline LF218 & BM005487 & $50 / 1.5$ & 176 & AAGGGCCCAGTTCGCATG/CCGGGTTCGATGTTGATCGA \\
\hline LF231 & BM005478 & $50 / 1.5$ & 100 & TTGCTGAAGGTGTCCAAGGA/TGTTGGACAGTTCCTCACGC \\
\hline LF250 & T58311 & $54 / 1.5$ & 152 & GTGGAGACTGGCCGCG/TCAGGTGCAGGTGGTTCAC \\
\hline LF253 & T58331 & $58 / 1.5$ & 214 & TGAAGACTCTCCGCCAGG/CGGATGATATCGGAATCT \\
\hline LF272 & BM005484 & $54 / 3.0$ & 147 & GTGCGTGGTATCTCCATCAA/GATGTTGTTGAAGTCCAGCTG \\
\hline LF291 & BM005482 & $54 / 1.5$ & 250 & GAACACCTTGGCCGAGTG/GTGCACAGGGAACCATCG \\
\hline LF334 & BM005506 & $54 / 1.5$ & 200 & GTTCATGAAGTACGCCAAGG/CTTGCCGATGCATTCTCC \\
\hline LF417 & BM005499 & $54 / 1.5$ & 228 & GAAGACCAGGAAGCTACGTGG/GACCAGCGACCACAGCTT \\
\hline
\end{tabular}

For LF178, we sequenced the PCR product obtained from one individual each from Ae. aegypti, Ar. subalbatus, and $D$. melanogaster. The CAT PCR products from Ar. subalbatus and D. melanogaster exhibited $92 \%$ and $83 \%$ nucleotide identity, respectively, when subjected to a pairwise alignment with the original Ae. aegypti LF178 cDNA sequence using the Blast2 program. ${ }^{32}$ A representative nucleotide and amino acid sequence alignment for all CAT PCR products based upon LF178, LF158, and LF103 is shown in Figure 3.

For CAT PCR products designed from Ae. aegypti LF158, we sequenced one individual each from Ae. aegypti and $D$. melanogaster. The $D$. melanogaster sequence was interesting in that it was 394 nucleotides in length while the Ae. aegypti PCR product was only 241 nucleotides in length. BLAST analysis of the D. melanogaster PCR product showed $100 \%$ nucleic acid identity and $100 \%$ amino acid identity to the Drosophila ribosomal protein L12 gene, GenBank accession number AAF47152, which contains four introns. The $D$. melanogaster CAT sequence spanned the fourth intron. The first exon in the D. melanogaster CAT PCR product showed $82 \%$ nucleotide identity with the original Ae. aegypti LF158 clone (nucleotides 1-135) and the second exon showed $74 \%$

A.

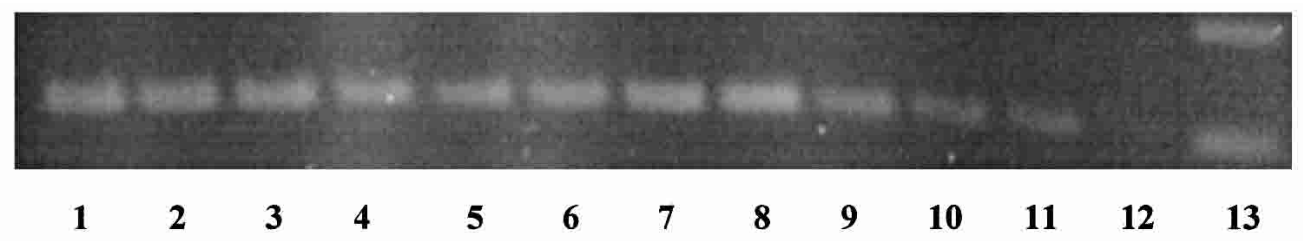

$300 \mathrm{bp}$

200 bp

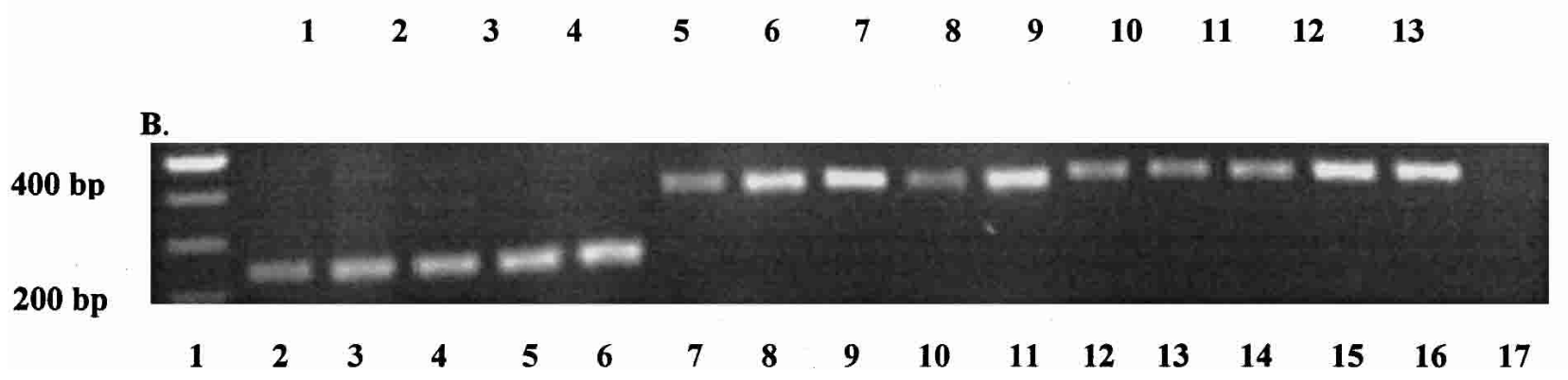

FIGURE 1. Representative agarose gels of single individual dipterans after amplification with comparative anchor-tagged sequences (CATS) polymerase chain reaction (PCR) primer pairs. A, Amplification products with the CATS LF178 primer pair. Lanes 1-3, products from single Aedes aegypti individuals; lanes 4-6, single Anopheles gambiae individuals; lanes 7 and 8, single Drosophila melanogaster individuals; lanes 9-11, single Culex pipiens individuals; lane 12, negative control lane; lane 13, 100-basepair molecular weight marker. B, Amplification products with the CATS LF158 primer pair. Lane 1, 100-basepair molecular weight marker; lanes 2-6, single Ae. aegypti individuals; lanes 7-11, single An. gambiae individuals; lanes 12-16, single D. melanogaster individuals; lane 17, negative control. The CAT LF158 PCR products from the An. gambiae and $D$. melanogaster individuals each contain an intron, while the product from the Ae. aegypti individuals does not. bp $=$ basepairs. 

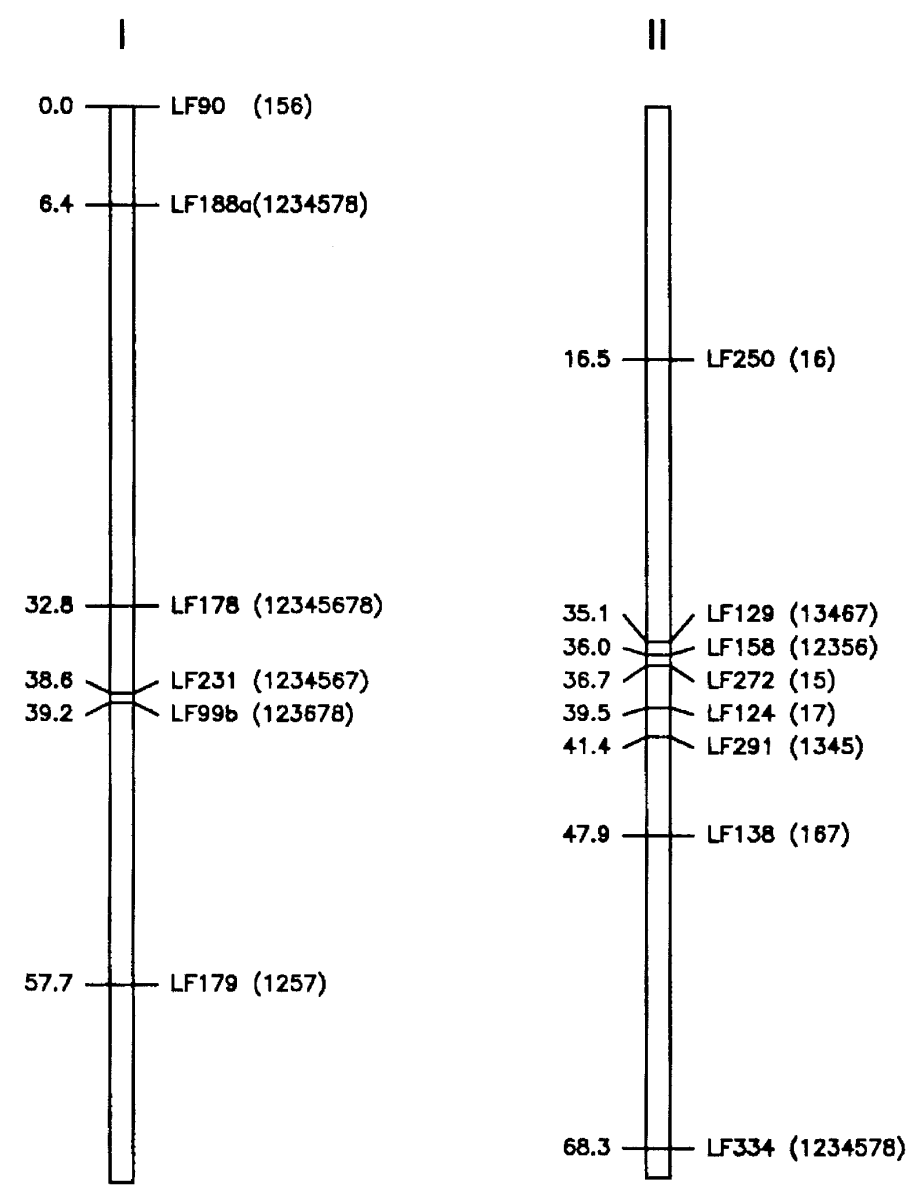

III

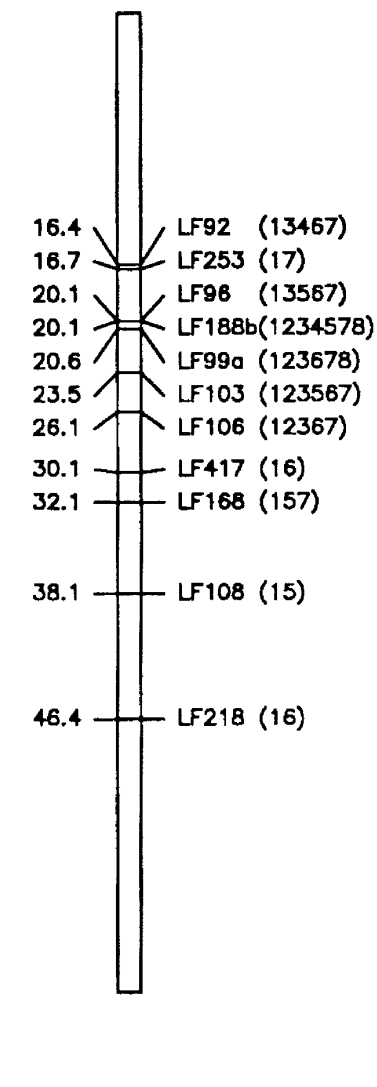

FIGURE 2. Relative distribution of comparative anchor-tagged sequence markers based on restriction fragment length polymorphism loci on the Aedes aegypti genetic map. The numbers in parentheses indicate the species amplified by that marker. $1=$ Ae. aegypti; $2=$ Drosophila melanogaster $; 3=$ Anopheles gambiae $; 4=$ Ae. togoi $; 5=$ Armigeres subalbatus $; 6=$ Culex pipiens; $7=$ Ochlerotatus triseriatus $; 8=$ Rhagoletis pomonella. I, II, and III refer to chromosome numbers. Map distances are listed in Kosambi centiMorgans.

nucleotide identity with the same clone sequence (nucleotides 289-394). The CAT PCR product obtained from An. gambiae also seemed to contain an intron (Figure 1B).

Finally, for LF103 we sequenced PCR products obtained from one individual each of Ae. aegypti and Ar. subalbatus. The Ar. subalbatus CAT sequence showed $88 \%$ nucleotide identity with the original Ae. aegypti LF103 cDNA when the two sequences were subjected to a pairwise sequence alignment using the Blast 2 computer program. ${ }^{32}$

TABLE 2

Comparative anchor-tagged sequence amplification success rate in Diptera

\begin{tabular}{llc}
\hline \multicolumn{1}{c}{ Genus and species } & \multicolumn{1}{c}{ Family } & \% single product* \\
\hline Aedes aegypti & Culicidae & 100 \\
Ochlerotatus triseriatus & Culicidae & 61 \\
Armigeres subalbatus & Culicidae & 52 \\
Anopheles gambiae & Culicidae & 48 \\
Culex pipiens & Culicidae & 48 \\
Drosophila melanogaster & Drosophilidae & 35 \\
Aedes togoi & Culicidae & 26 \\
Rhagoletis pomonella & Trypetidae & 13 \\
\hline * The percentage of the 23 primers listed in Table 1 that produced a single polymerase \\
chain reaction product.
\end{tabular}

\section{DISCUSSION}

Mosquitoes, members of the insect order Diptera and family Culicidae, are divided into three subfamilies: Culicinae, Anophelinae, and Toxoryhnchitinae. ${ }^{1}$ A limited number of species within each of the former two subfamilies serve as obligate intermediate vectors for a variety of diseases such as malaria, dengue fever, yellow fever, and lymphatic filariasis. Malaria alone accounts for 2.7 million deaths annually, lymphatic filariasis afflicts and disables upwards of 120 million people each year ${ }^{34}$ and dengue strikes 50 million individuals each year with an ever increasing number of accompanying cases of dengue hemorrhagic fever. ${ }^{34}$

The development of CATS markers with utility across a wide range of mosquito species could prove to be very useful for identifying species-specific as well as common genes involved in vector competence for malaria, filariasis, and arboviruses. That is, a comparative genomics approach facilitated by such markers would allow us to rapidly determine if the same genomic regions play a role in vector competence. Positional cloning efforts to isolate and characterize the individual genes from these regions could then be better directed toward mosquito species that are more fully characterized genetically and more amenable to laboratory manipulations because many of the mosquito species responsible for disease 


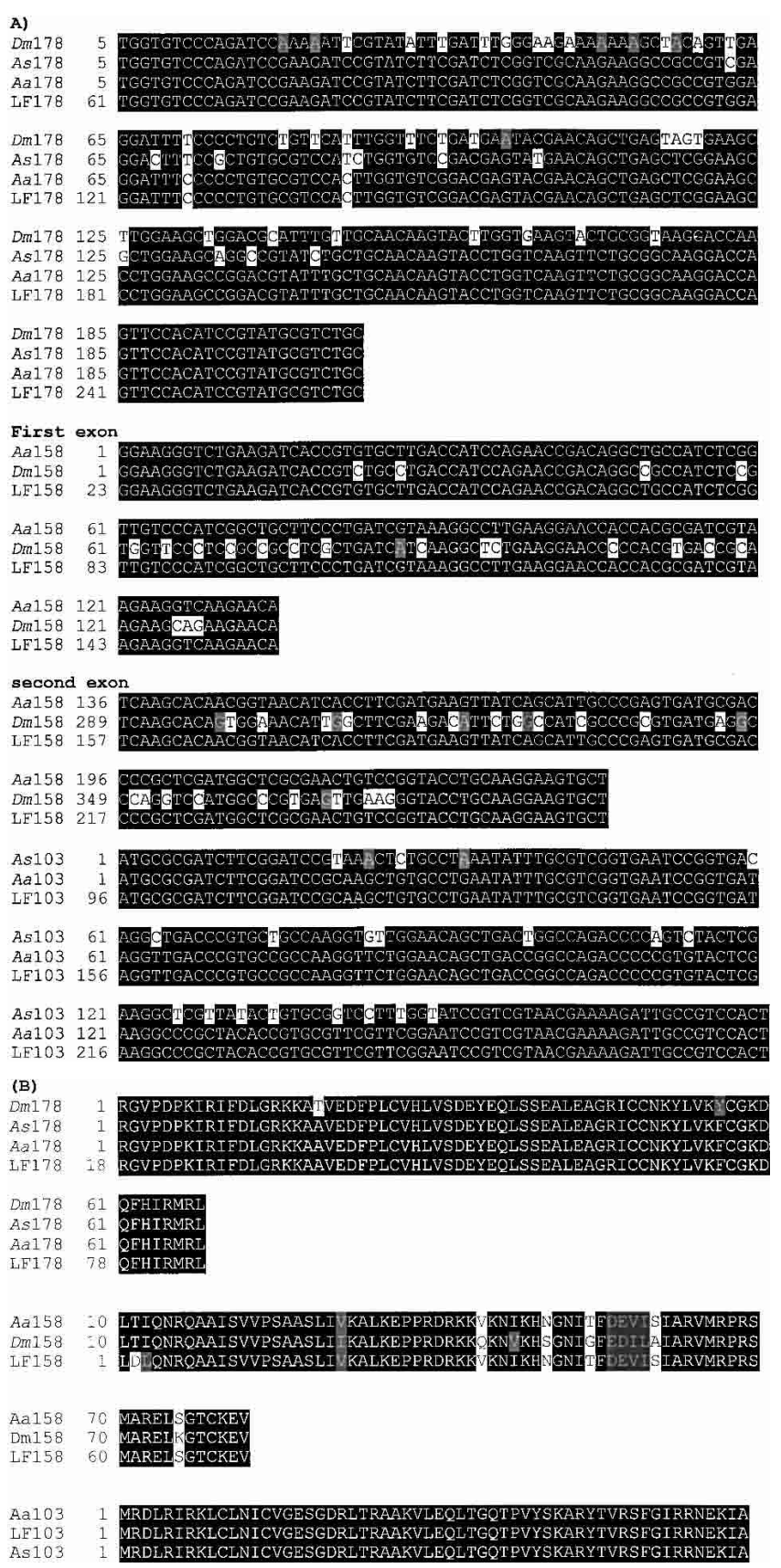

Figure 3. Nucleotide (A) and deduced amino acid (B) sequences from Aedes aegypti (Aa), Armigeres subalbatus (As), and Drosophila melanogaster (Dm), and LF178, 158, and 103 sequences from Ae. aegypti. Black shading signifies complete nucleotide or amino acid identity and similar amino acids are shaded in gray.

transmission to humans are extremely difficult to colonize in the laboratory or have never been successfully colonized. Indeed, genome regions containing quantitative trait loci (QTL) have previously been identified that are associated with vector competence of Aedes aegypti to Brugia malayi, a filariod nematode,${ }^{11,13}$ to Plasmodium gallinaceum, ${ }^{12}$ and to the dengue-2 virus. ${ }^{16}$ In $A n$. gambiae, QTL have been associated with encapsulation of $P$. cynomolgi $B$ and $P$. berghei. ${ }^{14,15}$

We have developed a PCR-based strategy for producing genetic markers that can be used as a comparative core set for rapid genetic mapping of multiple species within the Culi- cidae. We developed these markers by using a comparative genomics approach based upon CATS. Using available sequence data from Ae. aegypti ESTs and genes, we were able to develop 23 CATS primer pairs that generated a single-copy PCR product in Ae. aegypti and at least one of seven additional mosquito or more distantly related dipteran species. These markers exhibit broad genome coverage within $A e$. aegypti and thus broad potential coverage within other dipterans and clearly demonstrate the utility of a CATS-based strategy for marker development within the diptera.

Failure of the CATS primers to amplify a PCR product from genomic DNA was likely due to sufficient nucleotide divergence from the Ae. aegypti sequence, leading to the selection of primers within those regions of nucleotide divergence or to the presence of an intron. Interference by introns could include intron/exon boundaries residing within the selected primer sequence or due to instances in which an intron was too large to allow for amplification.

Our CATS markers can be easily applied to genetic studies in previously uncharacterized mosquito species. For example, the CAT PCR products can be rapidly screened for polymorphism and subsequent mapping using SSCP screening with subsequent silver staining. ${ }^{9,35}$ Single nucleotide polymorphisms can be identified within CATS and genotyping performed through the use of various techniques including melting curve analysis of single nucleotide polymorphisms. ${ }^{36}$

The development and subsequent mapping of CATS within the Diptera should also be useful in identifying syntenic relationships among species. Comparisons of linkage relationships of biochemical and morphologic markers suggested that linkage group conservation existed among the higher flies, Ceratitis capitata, D. melanogaster, and Musca domestica, but the relationship of mosquito linkage groups to these species was not clear. ${ }^{37}$ Recent reports within the Drosophila indicates that while syntenic regions are common, there has been extensive rearrangement of gene orders such that the estimated length of conserved chromosomal fragments between any two species of Drosophila is expected to be no greater than approximately 20-600 kilobases. ${ }^{38,39}$ A comparative genomic analysis of $D$. melanogaster and An. gambiae uncovered microsytenic blocks of 2-3 genes between these two species that were estimated to correspond to approximately 50-80-kilobase genomic segments. ${ }^{40}$ A further analysis of the full D. melanogaster and An. gambiae genomes revealed the presence of 948 microsyntenic blocks, with a microsytenic block being defined as having at least two orthologous groups within the region. ${ }^{41}$ The total fraction of Anopheles orthologs assigned to these microsyntenic blocks is $34 \%$ and constitutes a significant level of local conservation. ${ }^{41}$ Previously, six microsyntenic clusters composed of 17 orthologues between $A n$. gambiae and D. melanogaster were identified within the Pen1 region of $A n$. gambiae, thus demonstrating that gene arrangements in their last common ancestor persist in these two species to some degree. ${ }^{42}$ When 157 An. funestus cDNAs were physically mapped to polytene chromosomes of this species as well as mapped in silico (electronically, i.e., by sequence comparisons with gene databases) to An. gambiae, perfect preservation of synteny was observed but substantial shuffling of gene order was also seen. ${ }^{43}$ In contrast to this, comparisons within the Culicinae show conservation of gene order. ${ }^{19}$

The development of high-density CAT maps could provide an excellent means for eventually delineating the boundaries 
of syntenic regions ${ }^{23}$ and identifying regions of additional microsynteny, which may exist between dipterans in general and particularly among the great majority of mosquito species who are members of the sub-family Culicinae.

Additionally, CATS could prove valuable for phylogenetic studies. Using CATS, sequence comparisons can be made between a wide variety of mosquito species, which would contribute toward a greater understanding of systematic and taxonomic relationships between mosquitoes. It is believed that the lower diptera (mosquitoes, midges, gnats) diverged from the higher flies (Drosophila and house flies) about 250 million years ago. ${ }^{44}$ Traditional evolutionary systematics in mosquitoes was based on morphologic characters ${ }^{44}$ that placed the Anophelinae at the basal position, with Toxoryhnchitinae intermediate and Culicinae the most recently derived. ${ }^{44}$ Recent molecular data has supported this assertion, as shown by the use of the white gene by Besansky and Fahey $^{45}$ to evaluate taxonomic relationships among the Culicidae. It is interesting to note that our success in amplification with CATS primer pairs was highly correlated with the reported evolutionary relationship among the various species tested. Those species that had diverged more recently from Ae. aegypti, e.g., Oc. triseriatus and C. pipiens, exhibited greater success in amplification as CATS, while those that diverged earlier, e.g., An. gambiae, D. melanogaster, and $R$. pomonella, were less successful in amplifying with the CATS primer pairs.

Given that the total genome sequence is available for $D$. melanogaster, ${ }^{46}$ and just recently for An. gambiae, ${ }^{47}$ and an Ae. aegypti genome project has recently been initiated (Severson DW, unpublished data), the application of comparative genomics to other Culicidae as well as to the Diptera in general is likely to grow rapidly. ${ }^{48}$ Our demonstration of CATS utility for inter-specific comparisons should facilitate that process.

Received December 27, 2002. Accepted for publication April 3, 2003.

Financial support: This work supported by National Institute of Health grants AI-33127 and AI-34337.

Authors' address: Eric W. Chambers, Diane D. Lovin, and David W. Severson, Center for Tropical Disease Research and Training, Department of Biologic Sciences, University of Notre Dame, Notre Dame, IN 46556, Telephone 574-631-3826, Fax 574-631-7413, E-mail: Severson.1@nd.edu

\section{References}

1. Knight KL, Stone A, 1977. A Catalog of the Mosquitoes of the World (Diptera:Culicidae). Baltimore, MD: The Thomas Say Foundation.

2. Munstermann LE, Craig GB Jr, 1979. Genetics of Aedes aegypti: updating the linkage map. J Hered 70: 291-296.

3. O'Brien SJ, 1990. Genetic Maps: Locus Maps of Complex Genomes: Lower Eukaryotes. Fifth edition. Volume 3. Cold Spring Harbor, NY: Cold Spring Harbor Laboratory Press.

4. O'Brien SJ, 1993. Genetic Maps: Locus Maps of Complex Genomes: Lower Eukaryotes (33 Maps). Sixth edition. Volume 3. Cold Spring Harbor, NY: Cold Spring Harbor Laboratory Press.

5. Matthews TC, Munstermann LE, 1994. Chromosomal repatterning and linkage group conservation in mosquito karyotypic evolution. Evolution 48: 146-154.

6. Severson DW, Mori A, Zhang Y, Christensen BM, 1993. Linkage map for Aedes aegypti using restriction fragment length polymorphisms. J Hered 84: 241-247.

7. Severson DW, Meece JK, Lovin DD, Saha G, Morlais I, 2002.
Linkage map organization of expressed sequence tags and sequence tagged sites in the mosquito Aedes aegypti. Insect Mol Biol 11: 371-378.

8. Antolin MF, Bosio CF, Cotton J, Sweeney W, Strand MR, Black WC IV, 1996. Intensive linkage mapping in a wasp (Bracon hebetor) and a mosquito (Aedes aegypti) with single-strand conformation polymorphism analysis of random amplified polymorphic DNA markers. Genetics 143: 1727-1738.

9. Fulton RE, Salasek ML, DuTeau NM, Black WC IV, 2001. SSCP analysis of cDNA markers provides a dense linkage map of the Aedes aegypti genome. Genetics 158: 715-726.

10. Wang R, Kafatos FC, Zheng L, 1999. Microsatellite markers and genotyping procedures for Anopheles gambiae. Parasitol Today 15: 33-37.

11. Severson DW, Mori A, Zhang Y, Christensen BM, 1994. Chromosomal mapping of two loci affecting filarial worm susceptibility in Aedes aegypti. Insect Mol Biol 3: 67-72.

12. Severson DW, Thathy V, Mori A, Zhang Y, Christensen BM, 1995. Restriction fragment length polymorphism mapping of quantitative trait loci for malaria parasite susceptibility in the mosquito Aedes aegypti. Genetics 139: 1711-1717.

13. Beerntsen BT, Severson DW, Klinkhammer JA, Kassner VA, Christensen BM, 1995. Aedes aegypti: a quantitative trait locus (QTL) influencing filarial worm intensity is linked to QTL for susceptibility to other mosquito-borne pathogens. Exp Parasitol 81: 355-362.

14. Zheng L, Cornel AJ, Wang R, Erfle H, Voss H, Ansorge W, Kafatos FC, Collins FH, 1997. Quantitative trial loci for refractoriness of Anopheles gambiae to Plasmodium cynomolgi B. Science. 276: 425-428.

15. Gorman MJ, Severson DW, Cornel AJ, Collins FH, Paskewitz SM, 1997. Mapping a quantitative trait locus involved in melanotic encapsulation of foreign bodies in the malaria vector, Anopheles gambiae. Genetics 146: 965-971.

16. Bosio CF, Fulton RE, Salasek ML, Beaty BJ, Black WC IV, 2000. Quantitative trait loci that control vector competence for dengue-2 virus in the mosquito Aedes aegypti. Genetics 156: 687698.

17. Mori A, Severson DW, Christensen BM, 1999. Comparative linkage maps for the mosquitoes (Culex pipiens and Aedes aegypti) based on common RFLP loci. J Hered 90: 160-164.

18. Severson DW, Mori A, Kassner VA, Christensen BM, 1995. Comparative linkage maps for the mosquitoes Aedes albopictus and Ae. aegypti, based on common RFLP loci. Insect Mol Biol 4: 41-45.

19. Anderson JR, Grimstead PR, Severson DW, 2001. Chromosomal evolution among six mosquito species (Diptera: Culicidae) based upon shared restriction fragment length polymorphisms. Mol Phylogenet Evol 20: 316-321.

20. Mori A, Tomita T, Hidoh O, Kono Y, Severson DW, 2001. Comparative linkage map development and identification of an autosomal locus for insensitive acetylcholinesterase-mediated insecticide resistance in Culex tritaeniorhynchus. Insect Mol Biol 10: $197-203$.

21. Ferdig MT, Taft AS, Severson DW, Christensen BM, 1998. Development of a comparative genetic linkage map for Armigeres subalbatus using Aedes aegypti RFLP markers. Genome Res 8: 41-47.

22. Severson DW, Mori A, Zhang Y, Christensen BM, 1994. The suitability of restriction fragment length polymorphism markers for evaluating genetic diversity among and synteny between mosquito species. Am J Trop Med Hyg 50: 425-432.

23. Lyons LA, Laughlin TF, Copeland NG, Jenkins NA, Womack JE, O'Brien SJ, 1997. Comparative anchor tagged sequences (CATS) for integrative mapping of mammalian genomes. Nat Genet 15: 47-56.

24. Smith E, Shi L, Drummond P, Rodriguez L, Hamilton R, Powell E, Nahashon S, Ramlal S, Smith G, Foster J, 2000. Development and charaterization of expressed sequence tags for the turkey (Meleagris gallopavo) genome and comparative sequence analysis with other birds. Anim Genet 31: 62-67.

25. Mazzarella R, Montanaro V, Kere J, Reinbold R, Ciccodicola A, D'Urso M, Schlessinger D, 1992. Conserved sequence-tagged sites: a phylogenetic approach to genome mapping. Proc Natl Acad Sci USA 89: 3681-3685. 
26. Caetano AR, Lyons LA, Laughlin TF, O'Brien SJ, Murray JD, Bowling AT, 1999. Equine synteny mapping of comparative anchor tagged sequences (CATS) from human chromosome 5. Mamm Genome 10: 1082-1084.

27. Chen Z.-Q, Lautenberger JA, Lyons LA, McKenzie L, O'Brien SJ, 1999. A human genome map of comparative anchor tagged sequences. J Hered 90: 477-484.

28. Lyons LA, Kehler JS, O'Brien SJ, 1999. Development of comparative anchor tagged sequences (CATS) for canine genome mapping. J Hered 90: 15-26.

29. Altschul SF, Madden TL, Schäffer AA, Zhang J, Zhang Z, Miller W, Lipman DJ, 1997. Gapped BLAST and PSI-BLAST: a new generation of protein database search programs. Nucleic Acids Res 25: 3389-3402.

30. Smith RF, Wiese BA, Wojzynski MK, Davison DB, Worley KC, 1996. BCM Search Launcher-an integrated interface to molecular biology data base search and analysis services available on the World Wide Web. Genome Res 6: 454-462.

31. Severson DW, 1997. RFLP analysis of insect genomes. Crampton JM, Beard CB, and Louis C, eds. The Molecular Biology of Insect Disease Vectors: A Methods Manual. London: Chapman and Hall: $309-320$.

32. Tatusova TA, Madden TL, 1999. Blast 2 sequences - a new tool for comparing protein and nucleotide sequences. FEMS Microbiol Lett 174: 247-250.

33. van Ooijen JW, 1994. DrawMap: a computer program for drawing genetic linkage maps. J Hered 85: 66.

34. World Health Organization, 1997. Division of Control of Tropical Diseases. Available on the World Wide Web at URL http:// www.who.ch.

35. Hiss RH, Norris DE, Dietrich CH, Whitcomb RF, West DF, Bosio CF, Kambhampati S, Piesman J, Antolin MF, Black WC IV, 1994. Molecular taxonomy using single-strand conformation polymorphism (SSCP) analysis of mitochondrial ribosomal DNA genes. Insect Mol Biol 3: 171-182.

36. Akey JM, Sosnoski D, Parra E, Dios S, Hiester K, Su B, Bonilla C, Jin L, Shriver MD, 2001. Melting curve analysis of SNPs (McSNP): a gel free and inexpensive approach for SNP genotyping. Biotechniques 30: 358-367.

37. Weller GL, Foster GG, 1993. Genetic maps of the sheep blowfly Lucilia cuprina: linkage group correlations with other dipteran genera. Genome 36: 495-506.

38. Ranz JM, Caceres M, Ruiz A, 1999. Comparative mapping of cosmids and gene clones from a $1.6 \mathrm{Mb}$ chromosomal region of Drosophila melanogaster in three species of distantly related subgenus Drosophila. Chromosoma 108: 32-43.

39. Ranz JM, Casals F, Ruiz A, 2001. How malleable is the eukaryotic genome? Extreme rate of chromosomal rearrangement in the genus Drosophila. Genet Res 11: 230-239.

40. Bolshakov VN, Topalis P, Blass C, Kokoza E, della Torre A, Kafatos FC, Louis C, 2002. A comparative genomic analysis of two distant Diptera, the fruit fly, Drosophila melanogaster, and the malaria mosquito, Anopheles gambiae. Genet Res 12: 5766.

41. Zdobnov EM, von Mering C, Letunic I, Torrents D, Suyama M, Copley RR, Christophides GK, Thomasova D, Holt RA, Sub- ramanian GM, Mueller HM, Dimopoulos G, Law JH, Wells MA, Birney E, Charlab R, Halpern AL, Kokoza E, Kraft CL, Lai Z, Lewis S, Louis C, Barillas-Mury C, Nusskern D, Rubin GM, Salzberg SL, Sutton GG, Topalis P, Wides R, Wincker P, Yandell M, Collins FH, Ribeiro J, Gelbart WM, Kafatos FC, Bork P, 2002. Comparative genome and proteome analysis of Anopheles gambiae and Drosophila melanogaster. Science 298: 149-159.

42. Thomasova D, Ton LQ, Copley RR, Zdobnov EM, Wang X, Hong YS, Sim C, Bork P, Kafatos FC, Collins FH, 2002. Comparative genomic analysis in the region of a major Plasmodium-refractoriness locus of Anopheles gambiae. Proc Natl Acad Sci USA 99: 8179-8184.

43. Sharakhov IV, Serazin AC, Grushko OG, Dana A, Lobo N, Hillenmeyer ME, Westerman R, Romero-Severson J, Costantini C, Sagnon N, Collins FH, Besansky NJ, 2002. Inversions and gene order shuffling in Anopheles gambiae and A. funestus. Science 298: 182-185.

44. Ross HH, 1951. Conflict with Culex. Mosq News 11: 128-132.

45. Besansky NJ, Fahey GT, 1997. Utility of the white gene in estimating phylogenetic relationships among mosquitoes (Diptera:Culicidae). Mol Biol Evol 14: 443-454.

46. Myers EW, Sutton GG, Delcher AL, Dew IM, Fasulo DP, Flanigan MJ, Kravitz SA, Mobarry CM, Reinert KH, Remington KA, Anson EL, Bolanos RA, Chou HH, Jordan CM, Halpern AL, Lonardi S, Beasley EM, Brandon RC, Chen L, Dunn PJ, Lai Z, Liang Y, Nusskern DR, Zhan M, Zhang Q, Zheng X, Rubin GM, Adams MD, Venter JC, 2000. A whole-genome assembly of Drosophila. Science 287: 2196-2204.

47. Holt RA, Subramanian GM, Halpern A, Sutton GG, Charlab R, Nusskern DR, Wincker P, Clark AG, Ribeiro JM, Wides R, Salzberg SL, Loftus B, Yandell M, Majoros WH, Rusch DB, Lai Z, Kraft CL, Abril JF, Anthouard V, Arensburger P, Atkinson PW, Baden $\mathrm{H}$, de Berardinis $\mathrm{V}$, Baldwin D, Benes V, Biedler J, Blass C, Bolanos R, Boscus D, Barnstead M, Cai S, Center A, Chatuverdi K, Christophides GK, Chrystal MA, Clamp M, Cravchik A, Curwen V, Dana A, Delcher A, Dew I, Evans CA, Flanigan M, Grundschober-Freimoser A, Friedli L, Gu Z, Guan P, Guigo R, Hillenmeyer ME, Hladun SL, Hogan JR, Hong YS, Hoover J, Jaillon O, Ke Z, Kodira C, Kokoza E, Koutsos A, Letunic I, Levitsky A, Liang Y, Lin JJ, Lobo NF, Lopez JR, Malek JA, McIntosh TC, Meister S, Miller J, Mobarry C, Mongin E, Murphy SD, O'Brochta DA, Pfannkoch C, Qi R, Regier MA, Remington K, Shao H, Sharakhova MV, Sitter CD, Shetty J, Smith TJ, Strong R, Sun J, Thomasova D, Ton LQ, Topalis P, Tu Z, Unger MF, Walenz B, Wang A, Wang J, Wang M, Wang X, Woodford KJ, Wortman JR, Wu M, Yao A, Zdobnov EM, Zhang H, Zhao Q, Zhao S, Zhu SC, Zhimulev I, Coluzzi M, della Torre A, Roth CW, Louis C, Kalush F, Mural RJ, Myers EW, Adams MD, Smith HO, Broder S, Gardner MJ, Fraser CM, Birney E, Bork P, Brey PT, Venter JC, Weissenbach J, Kafatos FC, Collins FH, Hoffman SL, 2002. The genome sequence of the malaria mosquito Anopheles gambiae. Science 298: 129-149.

48. Kaufman TC, Severson DW, Robinson GE. 2002. The Anopheles genome and comparative insect genomics. Science 298: 97-98. 